\title{
Analytical Approaches to the Description of Optical Microresonator Devices
}

\author{
Manfred Hammer, Kirankumar R. Hiremath and Remco Stoffer \\ $\mathrm{MESA}^{+}$Research Institute, University of Twente, The Netherlands
}

\begin{abstract}
Optical ring resonators are commonly discussed on the basis of a frequency-domain model, that divides a resonator into coupler elements, ring cavity segments, and the straight port waveguides. We look at the assumptions underlying this model and at its implications, including remarks on reciprocity/symmetry arguments, the general power transfer characteristics, the resonance condition, the spectral distance and width of the resonances, the quantities that describe the resonator performance, and a few remarks about tuning. A survey of bend mode properties and a coupler description in terms of coupled mode theory fills the abstract notions of the model. As an example for devices that rely on a standing wave principle, in contrast to the traveling waves found in the microrings, we consider in less detail microresonators with square or rectangular cavity shapes. Also here a frequency domain coupled mode theory can be applied that opens up simple possibilities to characterize resonant configurations.
\end{abstract}

\section{INTRODUCTION}

The current research on microresonators as building blocks for large scale integrated optics [1] concentrates for a major part on devices with circular, ring- or disk-shaped cavities. During our participation in the project NAIS [2] we experienced that most experimental and theoretical discussions of optical microring resonators use notions derived from a quite intuitive frequency domain model. In Section 2 we try to clarify the assumptions that this "standard" model is based upon, and develop its implications. The reasoning follows more or less Refs. [3, 4], where we concretize the abstract parameterized expressions by a few characteristic (2D) examples for bend modes and coupler structures, seen as parts of the microresonator. The present model leads to a description of the interaction between the cavity and the straight waveguides in terms of a frequency domain coupled mode theory. Alternatively one can adopt a time domain viewpoint, where the resonance frequencies of an isolated cavity can be directly identified. See Ref. [5] for a discussion of these concepts.

While the interest in optical ring resonators dates back some time already [6], other resonator shapes have attracted attention only quite recently. The list includes elliptical $[7,8]$, rectangular $[9,10]$, as well as other, more irregular cavity shapes $[11,12]$. The rectangular variants are the subject of Section 3. In contrast to the microrings, where traveling waves establish the resonances and where reflections do usually not play a role, the rectangular resonators are based on a standing wave principle; reflections are essential for the operation (see e.g. Ref. [9] for details on these notions). Also here the basics of the device characteristics can be evaluated along a quite general, analytic frequency domain model [13], though not as explicit as in the case of the microrings. Examples of a bimodal resonance in a rectangular cavity and of the corresponding multimode facet illustrate these concepts. 

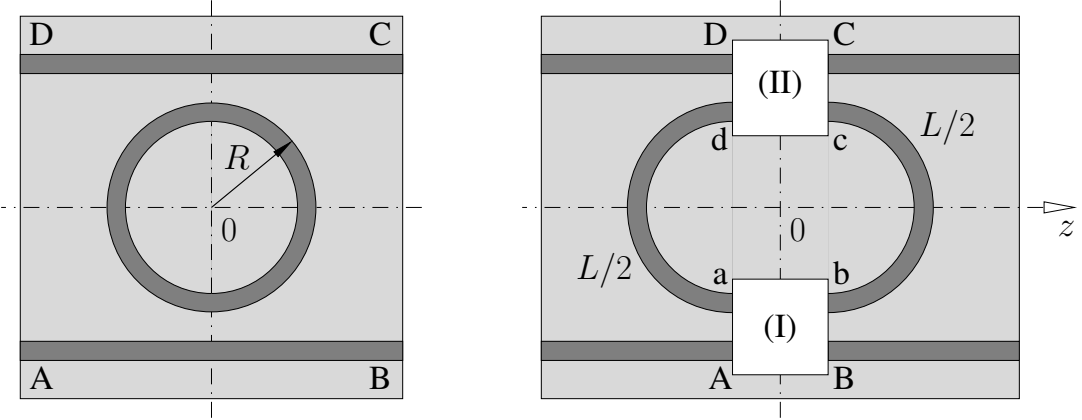

FIGURE 1. Schematic ring resonator representation (left) and the split configuration (right): Two identical directional couplers (I), (II) are connected by cavity waveguide segments of length $L / 2$. Letters A-D and a-d denote the coupler ports. The entire device has a twofold symmetry with respect to the centered horizontal and vertical planes. This implies that the four port waveguide segments are identical.

\section{2. “STANDARD MODEL” FOR OPTICAL RING RESONATORS}

Consider a ring resonator configuration as sketched in Figure 1. Two straight bus or port waveguides are evanescently coupled to the central cavity ring. If chiefly guided waves with reasonable confinement are present, one can expect that the interaction between the cavity and the port cores is localized around the two regions of closest approach. Hence, for purposes of modeling, the device is divided into these two coupler regions on the one hand, and the two parts of the cavity loop on the other hand. A prediction of the power transfer through the resonator requires a description of the light propagation along the two bent cavity waveguides segments, a model for what happens inside the coupler regions, and finally a framework to connect the parts. With some generality one can supply expressions with a few free parameters for the former two ingredients. Section 2.1 shows how the essentials of the ring resonator power transfer characteristic are evaluated within this parameterized model. The free parameters are eliminated in the subsequent paragraphs: Section 2.2 illustrates the basic modal properties of bend waveguides, Section 2.3 supplies an ansatz for the description of the coupler regions in terms of coupled mode theory (see Ref. [14] for further details on that subject). For simplicity, Sections 2.2-2.3 are restricted to two spatial dimensions.

\subsection{Abstract resonator model}

The "standard" model covers the propagation of light at fixed angular frequency $\omega=k \mathrm{c}$, usually specified by the vacuum wavelength $\lambda$, vacuum wavenumber $k=2 \pi / \lambda$, and vacuum speed of light $\mathrm{c}$. All optical fields vary in time according to $\sim \exp (\mathrm{i} \omega t)$. The list of underlying assumptions and approximations includes the following items:

- Single polarization operation is considered, none of the waveguide segments and coupler elements couples waves of different polarization; all waveguides are unimodal per polarization orientation. 
- The elements are purely linear and nonmagnetic. The various kinds of attenuation for light propagation along the cavity loops are incorporated in the modal properties, i.e. in the attenuation constant, of the cavity channel.

- Backreflections are negligible, inside the couplers as well as in the cavity loops. "Adiabatic" transitions along the light paths are required for the model to be valid.

- The interaction between the light paths is negligible outside the coupler regions. This assumption is likely to become violated by the long outer tails of bend or gallery modes in small, radiative cavities (see Section 2.2).

We now refer to the schematic splitting of the resonator as introduced in Figure 1. Variables $A_{ \pm}, B_{ \pm}, C_{ \pm}, D_{ \pm}$(external connections) and $a_{ \pm}, b_{ \pm}, c_{ \pm}, d_{ \pm}$(cavity connections) denote the amplitudes of the guided modes in the coupler port planes that are identified by the corresponding letters, where signs \pm identify waves that travel in the positive and negative $z$-direction. The operation of coupler I can be described by a scattering matrix that establishes a linear relation between the amplitudes $A_{-}, a_{-}, B_{+}, b_{+}$of the outgoing waves and the amplitudes $A_{+}, a_{+}, B_{-}, b_{-}$of the incoming fields,

$$
\left(\begin{array}{l}
A_{-} \\
a_{-} \\
B_{+} \\
b_{+}
\end{array}\right)=\left(\begin{array}{llll}
0 & 0 & \rho & \kappa \\
0 & 0 & \chi & \tau \\
\rho & \chi & 0 & 0 \\
\kappa & \tau & 0 & 0
\end{array}\right)\left(\begin{array}{l}
A_{+} \\
a_{+} \\
B_{-} \\
b_{-}
\end{array}\right),
$$

where the zeros implement the assumption of negligible backreflections. This matrix is symmetric due reciprocity arguments [15]. The reasoning involves basically an integration of a "reciprocity identity", valid for solutions of Maxwell's equations, over the coupler domain. Remaining boundary terms establish the symmetry relations between the field amplitudes on the input- and output ports of the circuit. The precise definition of the "ports" is crucial for the argument. Independent ports can be realized either by modal orthogonality (modes of differing order, or guided fields and surrounding radiation) or by spatially well separated outlets. The theorem holds for linear, nonmagnetic, potentially attenuating materials, in the presence of radiative losses, and irrespectively of the particular shape of the connecting cores. See Ref. [15] for details.

Due to the additional symmetry of the coupler element with respect to the vertical plane $z=0$ one can expect the transmission $A_{+} \rightarrow b_{+}$to be equal to the transmission $B_{-} \rightarrow a_{-}$. With a symmetric placement of the port planes and for identical mode profiles used for incident and outgoing waves, the corresponding entries $\kappa$ (lower left corner) and $\chi$ (second row, third column) of the scattering matrix in Eq. (1) must coincide $\kappa=\chi$ [15]. Coupler I transforms the mode amplitudes according to

$$
\left(\begin{array}{l}
A_{-} \\
a_{-}
\end{array}\right)=\left(\begin{array}{ll}
\rho & \kappa \\
\kappa & \tau
\end{array}\right)\left(\begin{array}{l}
B_{-} \\
b_{-}
\end{array}\right) \quad \text { and } \quad\left(\begin{array}{l}
B_{+} \\
b_{+}
\end{array}\right)=\left(\begin{array}{ll}
\rho & \kappa \\
\kappa & \tau
\end{array}\right)\left(\begin{array}{l}
A_{+} \\
a_{+}
\end{array}\right) .
$$

An analogous expression applies to coupler II, if the elements are identical:

$$
\left(\begin{array}{l}
D_{-} \\
d_{-}
\end{array}\right)=\left(\begin{array}{ll}
\rho & \kappa \\
\kappa & \tau
\end{array}\right)\left(\begin{array}{l}
C_{-} \\
c_{-}
\end{array}\right) \quad \text { and } \quad\left(\begin{array}{l}
C_{+} \\
C_{+}
\end{array}\right)=\left(\begin{array}{ll}
\rho & \kappa \\
\kappa & \tau
\end{array}\right)\left(\begin{array}{l}
D_{+} \\
d_{+}
\end{array}\right) .
$$


(The approximate constraint of lossless couplers requires $|\rho|^{2}+|\kappa|^{2}=1$ and $|\kappa|^{2}+$ $|\tau|^{2}=1$, consequently $|\rho|^{2}=|\tau|^{2}=1-|\kappa|^{2}$. Provided the input- and output planes are placed properly, one can even restrict to $\rho=\tau$. Note that these properties are not exploited in the following reasoning.)

Suppose the single mode ring waveguide segments of length $L / 2$ support the relevant cavity mode with complex propagation constant $\gamma=\beta-\mathrm{i} \alpha$, for phase propagation constant $\beta$ (real, positive) and attenuation constant $\alpha$ (real, positive, power attenuation constant: $2 \alpha$ ). For propagation along the cavity loop with $s$ measuring the propagation distance, the fields evolve according to $\sim \exp (-\mathrm{i} \gamma s)$, leading to the relations

$c_{-}=b_{+} \exp (-\mathrm{i} \beta L / 2) \exp (-\alpha L / 2), \quad a_{+}=d_{-} \exp (-\mathrm{i} \beta L / 2) \exp (-\alpha L / 2)$, and

$b_{-}=c_{+} \exp (-\mathrm{i} \beta L / 2) \exp (-\alpha L / 2), \quad d_{+}=a_{-} \exp (-\mathrm{i} \beta L / 2) \exp (-\alpha L / 2)$

of the mode amplitudes in the cavity port planes of the couplers.

\subsubsection{Power transfer}

Due to the linearity and the symmetry of the device it is sufficient to consider an excitation in only one of the external ports, say in port A. Given input amplitudes $A_{+}=\sqrt{P_{\mathrm{in}}}, B_{-}=D_{+}=C_{-}=0$, Eqs. (2)-(4) are to be solved for the directly transmitted power $P_{\mathrm{T}}=\left|B_{+}\right|^{2}$, and for the backwards dropped power $P_{\mathrm{D}}=\left|D_{-}\right|^{2}$, where neglecting reflections implies that there is no backreflected power $A_{-}=0$ and no power dropped in the forward direction $C_{+}=0$. This leads to the expressions

$$
D_{-}=\frac{\kappa^{2} p}{1-\tau^{2} p^{2}} A_{+}, \quad B_{+}=\left(\rho+\frac{\kappa^{2} \tau p^{2}}{1-\tau^{2} p^{2}}\right) A_{+},
$$

for the amplitudes in the drop- and through-port, where $p=\exp (-\mathrm{i} \beta L / 2) \exp (-\alpha L / 2)$. Splitting the cavity transfer coefficient $\tau$ of the coupler matrix as $\tau=|\tau| \exp (\mathrm{i} \varphi)$, and using the abbreviation $\tau-\kappa^{2} / \rho=d \exp (\mathrm{i} \psi)$, for real $d$ and $\psi$, one can write expressions

$$
P_{\mathrm{D}}=P_{\text {in }} \frac{|\kappa|^{4} \exp (-\alpha L)}{1+|\tau|^{4} \exp (-2 \alpha L)-2|\tau|^{2} \exp (-\alpha L) \cos (\beta L-2 \varphi)}
$$

for the dropped optical power and

$$
P_{\mathrm{T}}=P_{\text {in }} \frac{|\rho|^{2}\left(1+|\tau|^{2} d^{2} \exp (-2 \alpha L)-2|\tau| d \exp (-\alpha L) \cos (\beta L-\varphi-\psi)\right)}{1+|\tau|^{4} \exp (-2 \alpha L)-2|\tau|^{2} \exp (-\alpha L) \cos (\beta L-2 \varphi)}
$$

for the directly transmitted power. Note that here $L$ is the length of those parts of the cavity that are not already covered by the coupler model. In case of a ring with radius $R$, where each coupler region includes an arc length $l=\Delta \theta R$ of the cavity, one should evaluate the above expressions with $L=2 \pi R-2 \Delta \theta R$ (though one frequently encounters the approximation $L=2 \pi R$ corresponding to an interaction length that is short when compared to the cavity ring). Figure 2 shows a typical resonator spectrum as predicted by these expressions. Its features will be discussed in the following sections. 

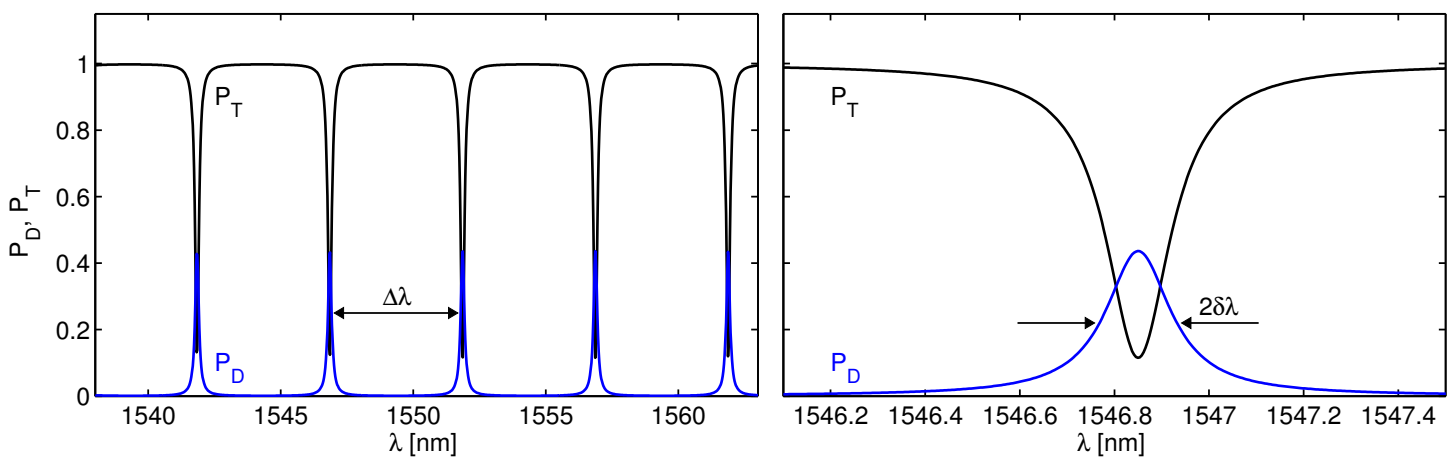

FIGURE 2. Wavelength dependence of the relative dropped and transmitted power fractions $P_{\mathrm{D}}$ and $P_{\mathrm{T}}$, for a $2 \mathrm{D}$ ring resonator with cavity segments according to Figure 4 and coupler regions as specified in Figure 7, for a cavity radius $R=50 \mu \mathrm{m}$, a gap $g=0.9 \mu \mathrm{m}$, and for TE polarized light. Characterizing quantities are the free spectral range $\Delta \lambda=5.01 \mathrm{~nm}$, the resonance width $2 \delta \lambda=0.17 \mathrm{~nm}$, a finesse of $F=30$, the quality factor $Q=9400$, and the power drop at resonance of 0.44 .

\subsubsection{Spectral response \& resonances}

Almost all quantities that enter expressions (5), (6), (7) must be assumed to be wavelength dependent. Hence the proper way to compute the resonator spectrum would be to evaluate the properties of the port waveguides, the cavity segments, and of the coupler regions as input for the above expressions, for a series of wavelengths.

A little more insight can be gained if one accepts the following approximation: If only a narrow wavelength interval needs to be considered, one can assume that the significant changes in $P_{\mathrm{D}}$ and $P_{\mathrm{T}}$ originate exclusively from the cosine terms that include the phase information. To account (approximately) for a nonnegligible length $l$ of the cavity segments in the coupler regions, we rewrite the phase term as $\beta L-2 \varphi=\beta L_{\text {cav }}-\phi$, with $L_{\text {cav }}$ being the complete cavity length, and $\phi=2 \beta l+2 \varphi$ (a corresponding procedure is applied to the phase term in the numerator of Eq. (7)). Further only the wavelength dependence of the phase propagation constant $\beta$ as it appears explicitly in the term $\beta L_{\text {cav }}-\phi$ is considered. In this way we incorporate the wavelength dependence of the phase gain $\beta L_{\mathrm{cav}}$ along the entire cavity, but disregard the wavelength dependence of the phase change $\phi$ that is introduced by the interaction with the port waveguides.

Resonances, maxima of the dropped power, are now characterized by singularities in the denominators of Eqs. (6), (7), i.e. by the condition $\cos \left(\beta L_{\mathrm{cav}}-\phi\right)=1$, or alternatively by the constraint

$$
\beta=\frac{2 m \pi+\phi}{L_{\mathrm{cav}}}=: \beta_{m} \quad \text { for integer } m .
$$

In case a resonant configuration is realized, the dropped power evaluates to

$$
\left.P_{\mathrm{D}}\right|_{\beta=\beta_{m}}=P_{\text {in }} \frac{|\kappa|^{4} \exp (-\alpha L)}{\left(1-|\tau|^{2} \exp (-\alpha L)\right)^{2}}
$$

Properly computed values for $\kappa$ and $\tau$ include already the losses along the parts of the cavity inside the couplers. Therefore $L$ in Eq. (9) (and in those places of Eqs. (6), (7) 
where attenuation is concerned) must not be replaced by $L_{\mathrm{cav}}$.

Using the approximations as introduced, evaluation of a resonator spectrum requires merely a single coupler simulation for a central wavelength, say $\lambda_{0}$, plus the wavelength dependence of the cavity phase propagation constant $\beta$, where again an approximation $\beta(\lambda)=\beta_{0}-\left(\beta_{0} / \lambda_{0}\right)\left(\lambda-\lambda_{0}\right)$ can be applied, motivated by Eqs. (11), (12). Figure 2 has been computed in this way.

\subsubsection{Free spectral range}

The resonant configuration next to a resonance found for $\beta_{m}$ is approximated as

$$
\beta_{m-1}=\frac{2(m-1) \pi+\phi}{L_{\mathrm{cav}}}=\beta_{m}-\frac{2 \pi}{L_{\mathrm{cav}}} \approx \beta_{m}+\left.\frac{\partial \beta}{\partial \lambda}\right|_{m} \Delta \lambda
$$

where $\Delta \lambda$ is the difference between the vacuum wavelengths corresponding to the two resonant configurations. By virtue of homogeneity arguments [16] for the propagation constant $\beta\left(\lambda, q_{j}\right)$, viewed as a function of the wavelength and all geometrical parameters $q_{j}$ that define the cavity waveguide cross section, one finds

$$
\frac{\partial \beta}{\partial \lambda}=-\frac{1}{\lambda}\left(\beta+\sum_{j} q_{j} \frac{\partial \beta}{\partial q_{j}}\right) \approx-\frac{\beta}{\lambda}
$$

for the wavelength dependence of the propagation constant in the cavity loop. Regarding the term in parentheses as an expansion of $\beta\left(\lambda, q_{j}+q_{j}\right)$, the sum can be neglected as a (crude) zeroth order approximation. The same (crude) approximation can be obtained if one rewrites the propagation constant $\beta=2 \pi n_{\mathrm{eff}} / \lambda$ in terms of vacuum wavenumber and effective mode index and neglects the wavelength dependence of the effective index:

$$
\frac{\partial \beta}{\partial \lambda}=-\frac{\beta}{\lambda}+k \frac{\partial n_{\mathrm{eff}}}{\partial \lambda} \approx-\frac{\beta}{\lambda} .
$$

This leads to the expression

$$
\Delta \lambda=-\left.\frac{2 \pi}{L_{\mathrm{cav}}}\left(\left.\frac{\partial \beta}{\partial \lambda}\right|_{m}\right)^{-1} \approx \frac{\lambda^{2}}{n_{\mathrm{eff}} L_{\mathrm{cav}}}\right|_{m}
$$

for the free spectral range (FSR) $\Delta \lambda$ of the device around the resonance of order $m$ that is associated with the wavelength $\lambda$ and the effective mode index $n_{\mathrm{eff}}=\lambda \beta_{m} /(2 \pi)$ of the cavity waveguide.

A more accurate and still simple expression results, if one does not introduce the approximations (11) or (12), i.e. if the wavelength derivative of $\beta$ or $n_{\text {eff }}$, respectively, is explicitly incorporated. Customarily one rewrites the left equality of (12) as

$$
\frac{\partial \beta}{\partial \lambda}=-\frac{k}{\lambda} n_{\mathrm{eff}, \mathrm{g}}
$$

where

$$
n_{\mathrm{eff}, \mathrm{g}}=n_{\mathrm{eff}}-\lambda \frac{\partial n_{\mathrm{eff}}}{\partial \lambda}
$$


is the group effective index of the cavity mode [4]. The expression for the free spectral range then reads

$$
\Delta \lambda=\frac{\lambda^{2}}{n_{\mathrm{eff}, \mathrm{g}} L_{\mathrm{cav}}} .
$$

\subsubsection{Spectral width of the resonances}

A configuration that drops about half of the maximum power is realized for a propagation constant $\beta+\delta \beta$ with $1 /\left(1+|\tau|^{4} \exp (-2 \alpha L)-2|\tau|^{2} \exp (-\alpha L) \cos \left(\beta L_{\text {cav }}-\phi\right)\right)=$ $2 /\left(1+|\tau|^{4} \exp (-2 \alpha L)-2|\tau|^{2} \exp (-\alpha L) \cos \left(\beta L_{\mathrm{cav}}+\delta \beta L_{\mathrm{cav}}-\phi\right)\right)$. Using the second order approximation of the cosine terms around a resonant cavity propagation constant, one obtains

$$
\delta \beta= \pm \frac{1}{L_{\mathrm{cav}}}\left(\frac{1}{|\tau|} \exp (\alpha L / 2)-|\tau| \exp (-\alpha L / 2)\right)
$$

for the shift in propagation constants that distinguishes configurations with the maximum and the half dropped power. By means of an approximation $\delta \beta \approx-\left(\beta_{m} / \lambda\right) \delta \lambda$ analogously to Eqs. (11), (12), Eq. (17) yields directly an expression

$$
2 \delta \lambda=\left.\frac{\lambda^{2}}{\pi L_{\mathrm{cav}} n_{\mathrm{eff}}}\right|_{m}\left(\frac{1}{|\tau|} \exp (\alpha L / 2)-|\tau| \exp (-\alpha L / 2)\right)
$$

for the full-width-at-half-maximum (FWHM) $2 \delta \lambda$ of the resonance of order $m$.

Provided that the effective group index $n_{\text {eff,g }}$ of the cavity mode is at hand, the expression can be improved by replacing $n_{\mathrm{eff}}$ by $n_{\mathrm{eff}, \mathrm{g}}$.

\subsubsection{Finesse and $Q$-factor}

The finesse $F$ of the resonator is defined as the ratio of the free spectral range and the width of a resonance found for a specific vacuum wavelength. With the FSR and the FWHM given by Eqs. (13), (18), in the present model the finesse evaluates to

$$
F=\frac{\Delta \lambda}{2 \delta \lambda}=\pi \frac{|\tau| \exp (-\alpha L / 2)}{1-|\tau|^{2} \exp (-\alpha L)} .
$$

Closely related is the Q-factor, here defined as the ratio of the operation wavelength and the resonance width:

$$
Q=\frac{\lambda}{2 \delta \lambda}=\pi \frac{n_{\mathrm{eff}} L_{\mathrm{cav}}}{\lambda} \frac{|\tau| \exp (-\alpha L / 2)}{1-|\tau|^{2} \exp (-\alpha L)}=\frac{n_{\mathrm{eff}} L_{\mathrm{cav}}}{\lambda} F .
$$

Assuming a circular resonator of radius $R$ with ring length $L_{\text {cav }}=2 \pi R$, one finds the expression

$$
Q=k R n_{\mathrm{eff}} F
$$

for the relationship between $\mathrm{Q}$ and finesse.

As before, the approximations according to Eqs. (11), (12) can be avoided by substituting the effective cavity mode index $n_{\text {eff }}$ in Eqs. (20), (21) by the effective group index $n_{\text {eff,g }}$ as defined in Eq. (15). 

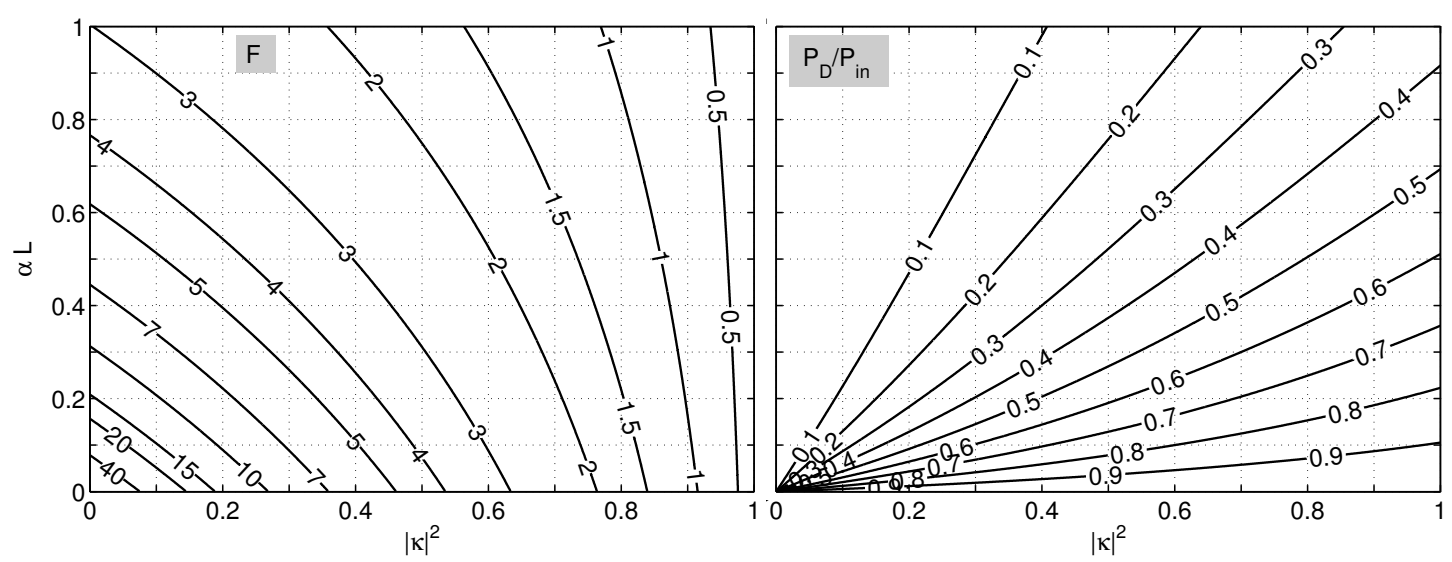

FIGURE 3. Contour levels of the finesse $F$ (left) and dropped power at resonance $P_{\mathrm{D}}$ (right) one the plane spanned by the squared coupling coefficient $|\kappa|^{2}$ (horizontal) and the cavity loss $\alpha L$ (vertical).

\subsubsection{Performance versus coupling strength \& losses}

Key quantities for an assessment of the performance of the ring resonator devices are the spectral width $2 \delta \lambda$ of the resonances, or the finesse $F$, on the one hand, and the maximum amount of power $P_{\mathrm{D}}$ that is dropped at resonance on the other hand. Assuming approximately lossless coupler elements $|\rho|^{2}=|\tau|^{2}=1-|\kappa|^{2}$, these quantities are given by

$$
F=\frac{\Delta \lambda}{2 \delta \lambda}=\pi \frac{\left(\sqrt{1-|\kappa|^{2}}\right) \exp (-\alpha L / 2)}{1-\left(1-|\kappa|^{2}\right) \exp (-\alpha L)}
$$

and

$$
\left.P_{\mathrm{D}}\right|_{\text {res }}=P_{\text {in }} \frac{|\kappa|^{4} \exp (-\alpha L)}{\left(1-\left(1-|\kappa|^{2}\right) \exp (-\alpha L)\right)^{2}},
$$

i.e. they are determined by the coupling constant $\kappa$ and the (logarithm of the) attenuation per round trip $\alpha L$ of the optical waves inside the cavity. Figure 3 shows explicit dependences of $F$ and $P_{\mathrm{D}}$ on these quantities for a general symmetric resonator device.

\subsubsection{Tuning}

Maxima of the dropped power are observed, if $\beta=(2 \pi m+\phi) / L_{\mathrm{cav}}=\beta_{m}$ holds for the propagation constant in the cavity waveguide, with integer order $m$ of the resonance. Assuming the wavelength dependence of the cavity propagation constant to be given, with $\beta_{m}$ a vacuum wavelength $\lambda_{m}=2 \pi n_{\mathrm{eff}, m} / \beta_{m}$ is associated such that $\beta\left(\lambda_{m}\right)=\beta_{m}$.

Disregarding its influence on the performance of the (short) couplers as a first approximation, a tuning mechanism, modeled by a small parameter $p$, affects mainly the light propagation along the cavity ring. Hence, besides on the wavelength, $\beta(p, \lambda)$ depends also on the tuning parameter, where $p=0$ represents the original state: $\beta\left(0, \lambda_{m}\right)=\beta_{m}$.

With the tuning applied, the resonance of order $m$ is shifted towards a new wavelength $\tilde{\lambda}_{m}$, such that $\beta\left(p, \tilde{\lambda}_{m}\right)=(2 \pi m+\phi) / L_{\mathrm{cav}}=\beta_{m}$ is satisfied again. A linear approximation 
in the tuning parameter and in the wavelength difference

$$
\beta\left(p, \tilde{\lambda}_{m}\right) \approx \beta\left(0, \lambda_{m}\right)+\left.p \frac{\partial \beta}{\partial p}\right|_{0, \lambda_{m}}+\left.\left(\tilde{\lambda}_{m}-\lambda_{m}\right) \frac{\partial \beta}{\partial \lambda}\right|_{0, \lambda_{m}} \stackrel{!}{=} \beta_{m}
$$

leads to an expression for the wavelength shift $\Delta_{p} \lambda_{m}=\tilde{\lambda}_{m}-\lambda_{m}$ that is effected by the tuning mechanism:

$$
\Delta_{p} \lambda_{m}=p \frac{\partial \beta}{\partial p} \frac{\lambda_{m}}{\beta_{m}} \quad \text { or } \quad \Delta_{p} \lambda_{m}=p \frac{\partial \beta}{\partial p} \frac{\lambda_{m}^{2}}{2 \pi n_{\mathrm{eff}, m}}
$$

i.e. the wavelength shift compensates the detuning of the propagation constants due to a nonzero perturbation strength $p$. Note that the wavelength shift does not depend on the length of the cavity.

As a more specific example we consider tuning of the resonator via the electrooptic effect. The optical permittivity of a material in the cavity region changes slightly, when an external static or quasistatic electric field is applied. Regarding the cavity waveguide locally to be adequately approximated by a straight channel with equal (two dimensional) cross section, one may apply the expressions known for the propagation constant shift due to small uniform permittivity perturbations [15]. Assume that application of the tuning field with (external) strength $E_{\mathrm{t}}$ alters the permittivity profile $\hat{\varepsilon}$ according to

$$
\hat{\varepsilon}\left(E_{\mathrm{t}}\right)=\hat{\varepsilon}(0)+E_{\mathrm{t}} \hat{e}
$$

where $\hat{\varepsilon}$ and $\hat{e}$ are functions of the cross section coordinates $x, y$ (here the spatial dependence of $\hat{e}$ includes the spatial distribution of materials and the spatial distribution of the static field strength). Then the propagation constant $\beta$ of a mode with electric part $\boldsymbol{E}=\left(E_{x}, E_{y}, \mathrm{i} E_{z}\right)$ and magnetic part $\boldsymbol{H}=\left(H_{x}, H_{y}, \mathrm{i} H_{z}\right)$ of the mode profile $\left(E_{x}-H_{z}\right.$ can be chosen real for a lossless structure) changes as

$$
\frac{\partial \beta}{\partial E_{\mathrm{t}}}=\frac{\omega \varepsilon_{0}}{2} \iint \boldsymbol{E}^{*} \cdot \hat{e} \boldsymbol{E} \mathrm{d} x \mathrm{~d} y / \iint\left(E_{x} H_{y}-E_{y} H_{x}\right) \mathrm{d} x \mathrm{~d} y .
$$

Using Eq. (25), one obtains

$$
\Delta_{E_{\mathrm{t}}} \lambda=E_{\mathrm{t}} \frac{\lambda}{2 n_{\mathrm{eff}}} \sqrt{\frac{\varepsilon_{0}}{\mu_{0}}} \iint \boldsymbol{E}^{*} \cdot \hat{e} \boldsymbol{E} \mathrm{d} x \mathrm{~d} y / \iint\left(E_{x} H_{y}-E_{y} H_{x}\right) \mathrm{d} x \mathrm{~d} y
$$

for the electrooptic wavelength shift of a resonance that is originally characterized by a wavelength $\lambda$ and effective cavity mode index $n_{\text {eff }}$.

Also here the approximations Eqs. (11), (12) enter. If available, the effective group index $n_{\text {eff,g }}$ according to Eq. (15) can replace the effective index $n_{\text {eff }}$ in Eqs. (25), (28). 


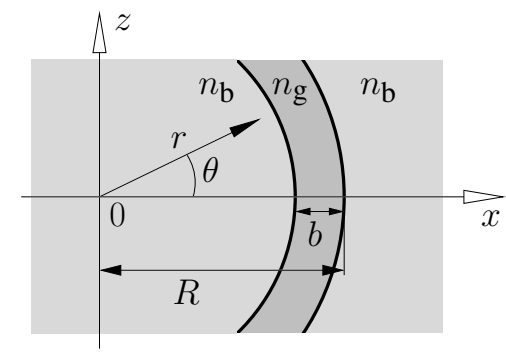

FIGURE 4. A bent slab waveguide. The core of thickness $b$ and refractive index $n_{\mathrm{g}}$ is embedded in a background medium with refractive index $n_{\mathrm{b}}$. The distance between the origin and the outer rim of the bend defines the bend radius $R$. Cartesian coordinates $x, z$ or alternatively polar coordinates $r, \theta$ will play a role. For the following simulations we use the default specification $n_{\mathrm{b}}=1.45$, $n_{\mathrm{g}}=1.6, b=1.0 \mu \mathrm{m}$.

\subsection{Modal properties of bent dielectric waveguides}

The former abstract model represents the resonator cavity as two pieces of a bent waveguide core. The model requires an expression for the relation of the guided wave amplitudes at the ends of segments with finite length. We thus have to look at the properties of modes that are supported by these waveguide bends [15, 17]. Figure 4 introduces a simple 2D geometry.

Being specified by the refractive index $n$ that depends on the radial coordinate $r$ (here $n(r)$ is piecewise constant), the bent waveguide can be seen as a structure that is homogeneous along the angular coordinate $\theta$. Hence one chooses an ansatz with pure exponential dependence on the azimuthal angle, analogous to the familiar case of a straight waveguide with homogeneity along the axis of propagation and a harmonic dependence on the propagation distance. For the bend, the angular propagation constant is commonly written as a product $\gamma R$, with a reasonably defined bend radius $R$. If multiplied by an angular interval $\Delta \theta$, one obtains the familiar combination of propagation constant $\gamma$ and propagation distance $R \Delta \theta$.

In case of a $2 D$ setting, where both the structure under consideration and the interesting solutions are constant along the $y$-coordinate, the ansatz for the electric and magnetic fields $\mathcal{E}, \mathcal{H}$ in the bend region reads:

$$
\left(\begin{array}{c}
\mathcal{E} \\
\mathcal{H}
\end{array}\right)(r, \boldsymbol{\theta}, t)=\frac{1}{2} \operatorname{Re}\left(\begin{array}{c}
\boldsymbol{E}_{\mathrm{b}}^{0} \\
\boldsymbol{H}_{\mathrm{b}}^{0}
\end{array}\right)(r) \mathrm{e}^{\mathrm{i} \omega t-\mathrm{i} \gamma R \boldsymbol{\theta} .}
$$

Here $\boldsymbol{E}_{\mathrm{b}}^{0}$ and $\boldsymbol{H}_{\mathrm{b}}^{0}$ are the electric and magnetic parts of the bend mode profile. For TE modes only the components $E_{\mathrm{b}, y}^{0}, H_{\mathrm{b}, r}^{0}$, and $H_{\mathrm{b}, \theta}^{0}$ are nonzero, while for TM fields only $H_{\mathrm{b}, y}^{0}, E_{\mathrm{b}, r}^{0}$, and $E_{\mathrm{b}, \theta}^{0}$ are present. $\gamma=\beta-\mathrm{i} \alpha$ is the complex valued propagation constant of the mode, with phase propagation constant $\beta$ and an imaginary part $-\alpha$ that accounts for the propagation losses (where additional loss mechanisms like material attenuation and scattering due to surface roughness are not included).

Upon inserting Eq. (29) into Maxwell's equations one arrives at the Bessel equation

$$
\frac{\mathrm{d}^{2} \phi}{\mathrm{d} r^{2}}+\frac{1}{r} \frac{\mathrm{d} \phi}{\mathrm{d} r}+\left(k^{2} n^{2}-\frac{\gamma^{2} R^{2}}{r^{2}}\right) \phi=0
$$

valid piecewise inside regions with constant refractive index $n$ for the basic field components $\phi=E_{\mathrm{b}, y}^{0}$ (TE) and $\phi=H_{\mathrm{b}, y}^{0}(\mathrm{TM})$. Eq. (30) is to be viewed as an eigenvalue problem in the angular propagation constant $\gamma R$. Eigenfunctions that are of interest here, 

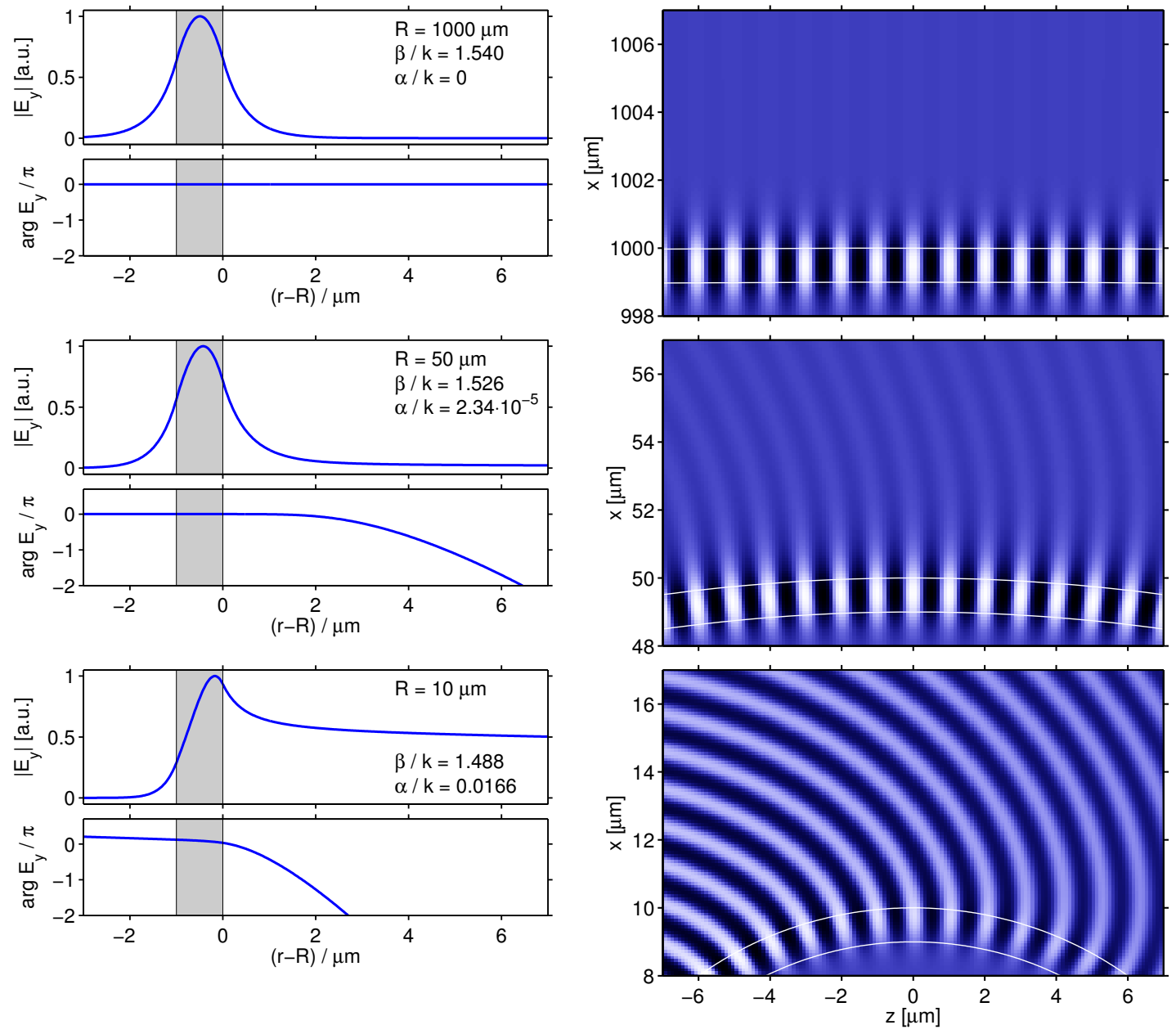

FIGURE 5. Fundamental TE modes of three bent slab waveguides with decreasing radii (top to bottom); plots of the mode profiles $E_{\mathrm{b}, y}^{0}$ (left) and snapshots of the propagating field $\mathcal{E}_{y}(x, z, t)$ at fixed time (right). Parameters are as specified in Figure 4 , for $\lambda=1.55 \mu \mathrm{m}$.

the bend modes, are nonzero solutions that remain bounded at the origin, that represent outgoing waves in the region $r>R$ (i.e. are given there by a Hankel function of second kind, cf. the sign of $\omega$ in Eq. (29)), with continuous quantities $\phi$ and $\mathrm{d}_{r} \phi$ (TE) or $\phi$ and $\left(\mathrm{d}_{r} \phi\right) / n^{2}(\mathrm{TM})$ at the interfaces. For details on the solution procedure and on the numerical implementation (in particular the evaluation of the required Bessel- and Hankel functions with large complex order turns out to be nontrivial) we refer to Ref. [18].

Figure 5 shows a series of typical bend mode profiles computed in this way. For large bend radii, one observes the familiar shape of a fundamental slab mode. With decreasing radius the mode profile maximum shifts towards the outer rim of the bent core. The plane phase front of the slab waveguide becomes more and more curved, leading to outwards traveling waves around the bend. These losses manifest by the growing imaginary part of the complex propagation constant. For small bend radii, the extension of the outer tail of the absolute value of the mode profile extends to radial distances that are substantially 

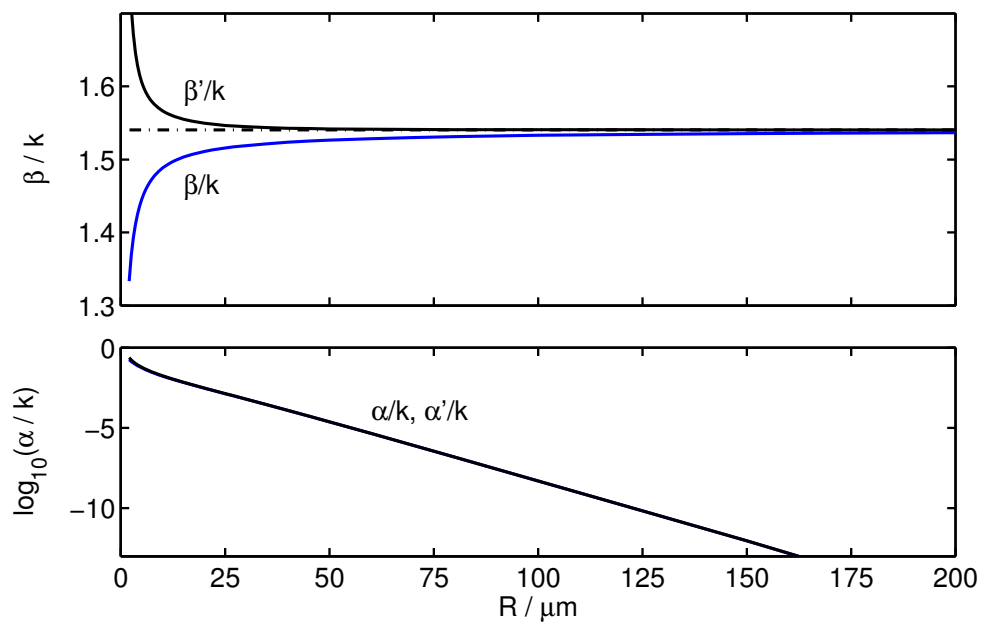

FIGURE 6. Dependence of phase and attenuation constants $\beta=k n_{\text {eff }}$ (top) and $\alpha$ (bottom) on the radius of bend slab waveguides according to Figure 4, for a wavelength of $1.55 \mu \mathrm{m}$. The horizontal line in the upper plot indicates the level of the effective index of a straight waveguide with equal width and refractive index profile. $\beta^{\prime}$ and $\alpha^{\prime}$ correspond to an alternative reference bend radius $R^{\prime}$, the distance from the origin to the core center.

larger than the width of the original straight slab mode. The field strength still converges to zero, but $\phi$ itself is not square integrable, according to the asymptotic expansions for the relevant Hankel functions.

The bend radius $R$ is commonly used as a measure for the curvature of the bend. According to Figure 6, with growing $R$ the levels for the phase and attenuation constants converge to the values of a slab waveguide with equal cross section. It must be emphasized here that the definition of $R$ is entirely arbitrary. Positioning $R$ at the outer rim of the ring rather than at the core center has the advantage that the definition is also applicable for disk configurations, where the inner boundary is absent.

Note that the values for the complex propagation constant $\gamma$ depend on the choice of the bend radius. The bend mode ansatz (29) and the eigenvalue equation (30) specify a unique angular propagation constant, the product $\gamma R$. With an alternative definition $R^{\prime}=R-b / 2$ of a bend radius at the core center, the expressions should lead to the same physical field, i.e. must result in the same angular phase constant $\gamma^{\prime} R^{\prime}=\gamma R$, hence we have a relation $\gamma^{\prime}=\gamma R /(R-b / 2)$ for the propagation constants emerging from the two choices for the bend radius. Certainly no physical reasoning should rely on this arbitrariness in the definition of $R$. This concerns e.g. statements about the growth or decay of phase propagation constants with $R$ (depending on the configuration the sign of the slope can indeed differ), or discussions about "phase matching" of bend waveguides with straight cores in a coupler configuration. However, care must be taken that the expressions of Section 2.1 are applied with parameters $\gamma, \beta, \alpha$, that fit to the definition of $R, L$, or $L_{\mathrm{cav}}$, respectively.

In contrast to discretized approximations derived by numerical (FD, FEM) means on a limited computational window, the present analytical solutions are defined on the entire positive $r$-axis. This turns out to be a significant advantage, if these profiles are to be used as basis fields in a coupled mode framework, as we will do in the following section. 


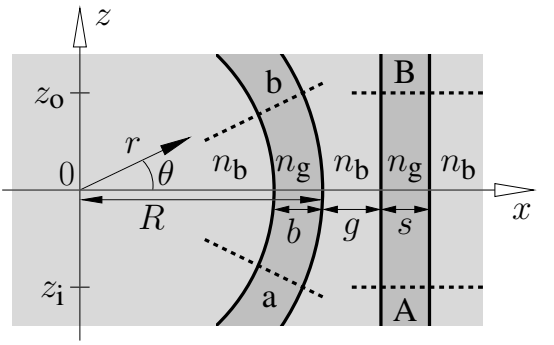

FIGURE 7. A coupler configuration. The core of a straight waveguide (thickness $s$, refractive index $n_{\mathrm{g}}$ ) is placed next to the bend of Figure 4, with a gap of width $g$ in between. Roman letters A, B, a, b indicate the ports of the coupler, as introduced in Section 2. Additional default parameter for the following simulations is the value $s=1.0 \mu \mathrm{m}$.

\subsection{Coupler modeling}

With the cavity properties being given in terms of the bend mode propagation constants, as a last ingredient the expressions of the "standard model" in Section 2.1 require the coupler scattering matrices, represented by the transfer coefficients $\rho$ and $\tau$ and by the coupling coefficient $\kappa$. Since we can now assume that the bend mode profiles and much more standard - mode profiles for the straight port waveguides are at hand, a natural way to proceed is to combine these profiles into a coupler description in terms of coupled mode theory.

Manifold variants of coupled mode theory have been proposed, for a variety of different domains of applications [19, 15, 20, 4]. Among these several papers (see e.g. $[21,22,23,24,25,26,27])$ deal with the evanescent interaction of waves in bent and straight waveguides; these differ in particular in the way the cavity fields (frequency domain bend modes or time domain gallery resonances) are computed and/or approximated, and in the soundness of the heuristics that are applied in the derivation and solution of the coupled mode equations. The formulation given below is based on a variational or reciprocity technique $[28,15]$ and an own implementation for straight waveguides [29]. One should emphasize that we are interested here not in an abstract model including fit parameters, but in an effective (and accurate) tool for ab-initio design.

Figure 7 introduces the relevant 2D coupler geometry. One assumes that the interaction is such that locally the electromagnetic field is accurately represented by a superposition of the profiles that correspond to the two individual cores. The interaction of the two basis fields is modeled by allowing the coefficients in the superposition to vary with the propagation distance. These notions lead to the following ansatz for the total electromagnetic field, written in Cartesian coordinates:

$$
\left(\begin{array}{c}
\mathcal{E} \\
\mathcal{H}
\end{array}\right)(x, z, t)=\frac{1}{2} \operatorname{Re}\left\{A_{\mathrm{b}}(z)\left(\begin{array}{c}
\boldsymbol{E}_{\mathrm{b}} \\
\boldsymbol{H}_{\mathrm{b}}
\end{array}\right)(x, z)+A_{\mathrm{s}}(z)\left(\begin{array}{c}
\boldsymbol{E}_{\mathrm{s}} \\
\boldsymbol{H}_{\mathrm{s}}
\end{array}\right)(x, z)\right\} \mathrm{e}^{\mathrm{i} \omega t} .
$$

Here

$$
\left(\begin{array}{c}
\boldsymbol{E}_{\mathrm{b}} \\
\boldsymbol{H}_{\mathrm{b}}
\end{array}\right)(x, z)=\left(\begin{array}{c}
\boldsymbol{E}_{\mathrm{b}}^{0} \\
\boldsymbol{H}_{\mathrm{b}}^{0}
\end{array}\right)(r(x, z)) \mathrm{e}^{-\mathrm{i} \gamma_{\mathrm{b}} R \theta(x, z)} \quad \text { and } \quad\left(\begin{array}{c}
\boldsymbol{E}_{\mathrm{s}} \\
\boldsymbol{H}_{\mathrm{s}}
\end{array}\right)(x, z)=\left(\begin{array}{c}
\boldsymbol{E}_{\mathrm{s}}^{0} \\
\boldsymbol{H}_{\mathrm{s}}^{0}
\end{array}\right)(x) \mathrm{e}^{-\mathrm{i} \beta_{\mathrm{s}} z}
$$

are the (known) profiles of the fundamental modes of the involved bend and straight waveguides, multiplied by the appropriate exponential dependences on the propagation constants $\gamma_{\mathrm{b}}=\beta_{\mathrm{b}}-\mathrm{i} \alpha_{\mathrm{b}}$ and $\beta_{\mathrm{s}}$, respectively. 
The subsequent procedure is based on the assumption that the ansatz (31) represents indeed a proper solution of Maxwell's equations for the coupler region. Thus we have solutions for the permittivity distribution of the isolated straight waveguide $\varepsilon_{\mathrm{s}}(x)$ at hand, one solution for the isolated bend waveguide $\varepsilon_{\mathrm{b}}(x, z)$ at hand, and a template for the full problem, specified by the permittivity $\varepsilon(x, z)$. For solutions of Maxwell's equations for two different permittivity distributions an integral identity commonly called the "Lorentz reciprocity theorem" can be derived straightforward [15]. Applying this identity to our basis fields and the template reduces the ansatz (31) to a set of coupled ordinary differential equations for the unknown mode amplitudes

$$
\left(\begin{array}{cc}
\sigma_{\mathrm{bb}} & \sigma_{\mathrm{bs}} \\
\sigma_{\mathrm{sb}} & \sigma_{\mathrm{ss}}
\end{array}\right) \frac{\mathrm{d}}{\mathrm{d} z}\left(\begin{array}{c}
A_{\mathrm{b}} \\
A_{\mathrm{s}}
\end{array}\right)=\left(\begin{array}{cc}
c_{\mathrm{bb}} & c_{\mathrm{bs}} \\
c_{\mathrm{sb}} & c_{\mathrm{ss}}
\end{array}\right)\left(\begin{array}{c}
A_{\mathrm{b}} \\
A_{\mathrm{s}}
\end{array}\right)
$$

with $z$-dependent (power-) coupling coefficients

$$
\begin{aligned}
\sigma_{p q} & =\frac{1}{4} \int\left(E_{p, x}^{*} H_{q, y}-E_{p, y}^{*} H_{q, x}+H_{p, y}^{*} E_{q, x}-H_{p, x}^{*} E_{q, y}\right) \mathrm{d} x, \\
c_{p q} & =-\mathrm{i} \frac{\omega \varepsilon_{0}}{4} \int \boldsymbol{E}_{p}^{*} \cdot\left(\varepsilon-\varepsilon_{q}\right) \boldsymbol{E}_{q} \mathrm{~d} x, \quad p, q=\mathrm{b}, \mathrm{s} .
\end{aligned}
$$

By numerical evaluation Eqs. (33) are finally solved for the unknown functions $A_{\mathrm{b}}$ and $A_{\mathrm{s}}$. The procedure consists of the numerical integration of the differential equation along a $z$-interval that represents the coupler region, and of the numerical quadrature of the integrals along $x$ on a suitable lateral computational window, for a series of discrete $z$ positions. Due to the linearity of the system, this procedure can be formulated directly for the transfer matrix that relates the amplitudes at the exit plane of the coupler to the values at the input plane. After projection on the slab mode profiles and a correction for the phase gain, one obtains directly the required coupler scattering matrix. For details concerning the implementation of this approach, concerning the precise evaluation of the scattering matrix, and for further results we refer to Ref. [14].

Besides the ansatz (31), the reasoning in terms of the reciprocity identity that leads to the system of coupled mode equations, and the approximations that enter the numerical solution of Eqs. (33), no further heuristics are applied in the present formulation (the use of the reciprocity theorem with the unidirectional ansatz of only forward propagating waves hides the neglect of second order derivatives, as is commonly seen in the derivation of coupled mode equations, cf. e.g. the detailed discussion Ref. [20]). Ref. [14] includes a comparison with rigorous numerical simulations, intended as a justification of the nevertheless rather arbitrary assumptions underlying Eq. (31).

Figure 8 displays three field examples obtained with the procedure outlined above. The configuration at the top corresponds to the resonator simulation of Figure 2. Reducing the separation of the two cores leads to an increased power transfer in the center coupler. If additionally the radius of the bent core is increased (bottom), one arrives at a configuration with a coupling coefficient $|\kappa|^{2}$ that is almost equal to the one of the structure at the top, i.e. the couplers effect similar levels of power transfer between the straight and the bend waveguide, despite the fact that the strength as well as the length of the interaction is much larger in the bottom configuration with larger bend radius and 


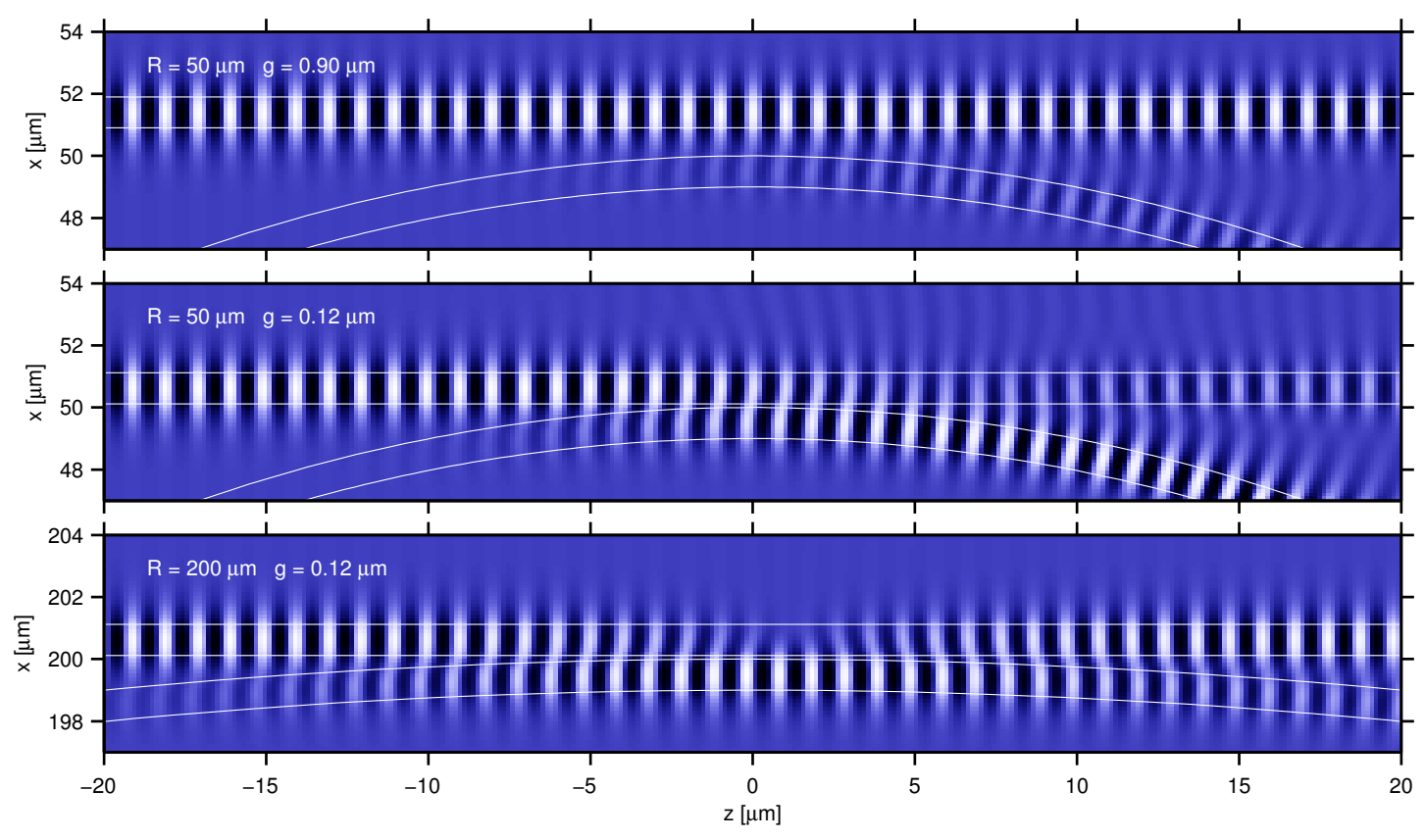

FIGURE 8. Coupled mode propagation along coupler configurations according to Figures 4 , 7, for $R=50 \mu \mathrm{m}, g=0.9 \mu \mathrm{m}$ (top), for $R=50 \mu \mathrm{m}, g=0.12 \mu \mathrm{m}$ (center), and for $R=200 \mu \mathrm{m}, g=0.12 \mu \mathrm{m}$ (bottom); TE polarized waves at a wavelength $\lambda=1.55 \mu \mathrm{m}$. The plots show snapshots of the physical electric field $\mathcal{E}_{y}(x, z, t)$ at a fixed time, for a coupler excitation by the guided wave of the straight waveguide in the upper left port. Absolute squares of the corresponding scattering matrix entries are $\left(|\rho|^{2},|\kappa|^{2},|\tau|^{2}\right)=(0.93,0.07,0.92)$ (top), $(0.16,0.83,0.16)$ (center), and $(0.93,0.07,0.93)$ (bottom).

smaller gap width. Clearly one has to distinguish between the magnitude of the matrix entries $c_{\mathrm{bs}}$ and $c_{\mathrm{sb}}$ in the differential equations (33), which represent the local interaction strength, and the offdiagonal coefficients $\kappa$ of the coupler scattering matrix, which enter the expressions in Section 2.1. These latter correspond to the solutions of Eqs. (33), and represent the net effect of the coupler.

We experienced that present discussions about coupler performance in ring resonator designs focus mainly on two issues. These are the influence of the separation between the cavity and the port waveguides on the one hand, and the "phase matching" of port and cavity cores on the other hand. Figure 9 shows the corresponding dependences of the net coupling coefficient $|\kappa|^{2}$ on the gap width $g$ and on the width $s$ of the port waveguide, for a series of configurations with our sample parameter set.

As expected, in general the net power transfer between the straight and the bend core grows with decreasing gap width, i.e. with increasing interaction strength, and with increasing bend radius, i.e. with increasing interaction length. These tendencies continue until maxima are reached, and are reversed afterwards. According to Figure 8, the reversal is caused by power fractions that are transfered from the straight core to the ring first, and that are then coupled back to the straight waveguide again, similar to the familiar periodic coupling process in two parallel straight waveguides. Just as in that setting, here the maximum levels of transfered power decrease, if the coupled systems become more and more different, as is the case for decreasing bend radius. 

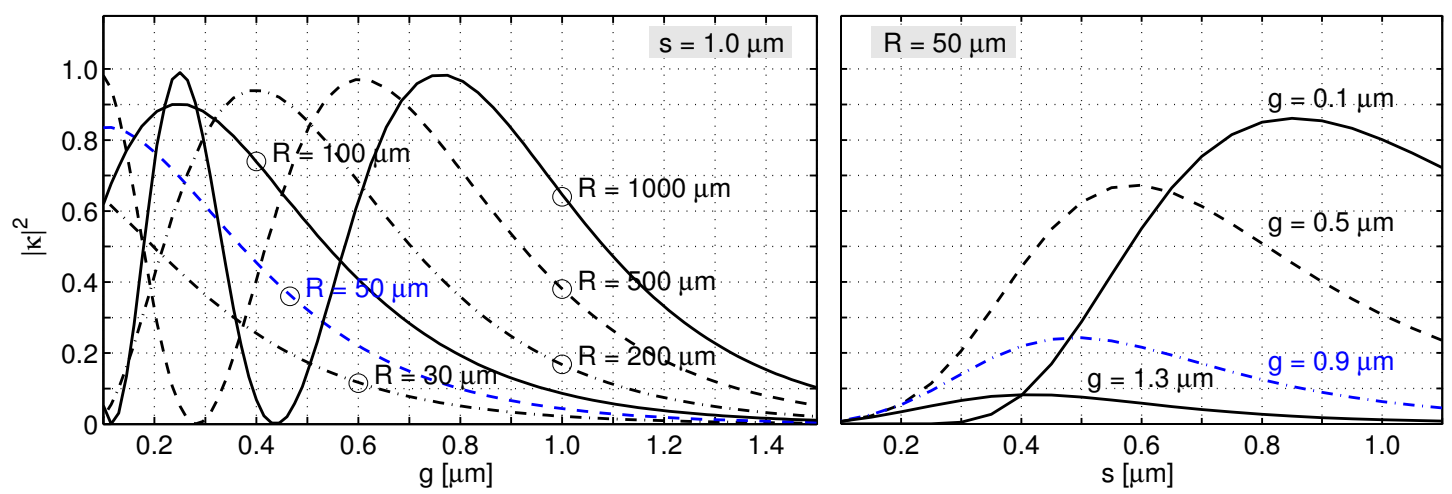

FIGURE 9. Coupling constants $|\kappa|^{2}$ for coupler configurations as introduced in Figure 7. The left plot shows the dependence on the gap width $g$, for different bend radii $R$ and a common thickness $s=1.0 \mu \mathrm{m}$ of the straight waveguide. The curves on the right illustrate the influence of the width of the straight channel, for different values of the gap width at a common bend radius $R=50 \mu \mathrm{m}$.

Concerning "phase matching", the right plot in Figure 9 does not indicate that a sensitive condition on the phase propagation constants exists that enables or prohibits proper coupler operation. Apart from the arbitrariness in the definition of $R$ and $\beta_{\mathrm{b}}$ (cf. Section 2.2, here one could think of evaluating $\beta_{\mathrm{b}}$ for different $R$ around the straight core), it would be difficult to separate the effect of the phase mismatch from the result of the change in the mode overlaps. According to Figure 3, a microresonator design with high finesse requires a coupling coefficient $|\kappa|^{2}$ below 10\%. For our parameter set with $R=50 \mu \mathrm{m}$, this should be achievable for any width of port waveguides in the interval $[0.3,1.1] \mu \mathrm{m}$, provided that the gap $g$ is properly adjusted.

Things are more clear in the limit of large resonators, where one has to deal basically with adiabatic straight directional couplers. Here the ratio between the mismatch of propagation constants and the coupling strength determines an upper limit for the transfer of power from one waveguide to another. This limitation is already visible in the curves in the left inset of Figure 9. A suitable phase mismatch, together with a properly selected gap width, could thus be employed to design a coupler that operates close to a (low level) maximum in $|\kappa|^{2}(g)$, i.e. to design a microresonator that is fault-tolerant with respect to changes in the width of the coupler gap.

\section{RECTANGULAR MICRORESONATORS}

For the remainder of this paper we shall have a look at a microresonator concept, where a dielectric rectangle replaces the former ring cavity. Figure 10 introduces the geometry. As before the cavity is evanescently coupled to the port waveguides, now with a constant gap distance. Hence one can expect an interaction along the full length of the cavity. The two facets at the ends of the cavity segment cause strong reflections of the waves that are excited inside the cavity, while one can assume that they have much less influence on the waves that travel along the port cores. These notions lead to the splitting as hinted at in 

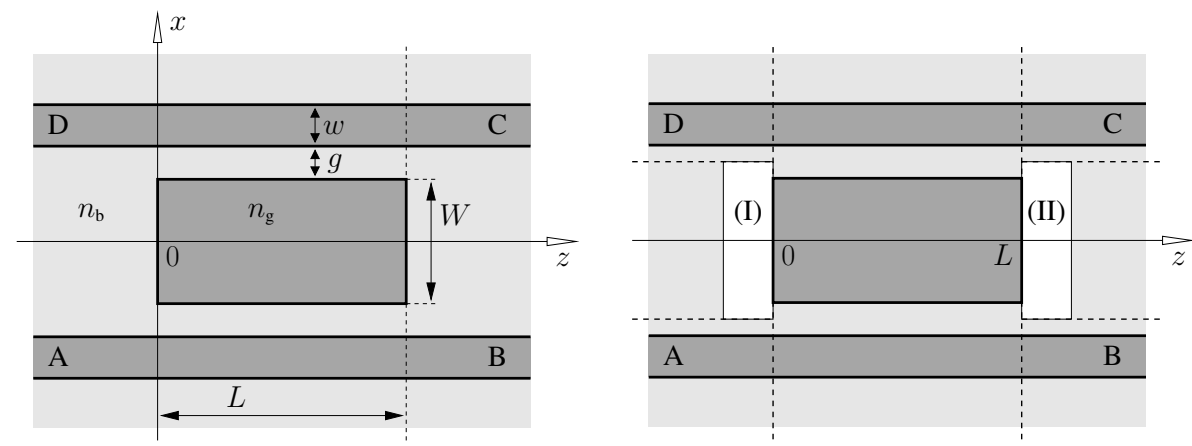

FIGURE 10. Geometry of the rectangular microresonators (left). Two parallel waveguides of width $w$ are separated by a gap $g$ from the rectangular cavity of width $W$ and length $L$. The core regions with refractive index $n_{\mathrm{g}}$ are embedded in a background medium with refractive index $n_{\mathrm{b}}$. Letters A to D identify the input respectively output ports of the device. For modeling purposes, the device is split into the central cavity segment $0<z<L$ and two facet regions I, II (right).

Figure 10: The resonator is divided into the cavity segment on the one hand and the two facet regions on the other hand. Different from the ring resonators, for the rectangular cavities reflections are essential. Consequently waves that travel in both the positive and negative $z$-directions have to be taken into account.

One can write quite general equations for what happens in the central coupler segment and for the effects of the facets. Upon accepting the approximation that the waves in the regions of the port waveguides are not influenced by the facet discontinuity and that the presence of the port cores can be neglected for the facet modeling, these equations can be combined into a parameterized resonator model. Due to the number of prospectively contributing basis fields this becomes a formulation in terms of propagation and reflection matrices. Explicit analytical evaluation should be possible, but tedious, hence we restrict to numerical examples. Just as for the ring resonators we consider the propagation of light with fixed frequency respectively vacuum wavelength; alternatively time-domain descriptions can be applied as well (cf. the proposal in Ref. [9], based on time-domain coupled mode equations and finite-difference-time-domain simulations).

\subsection{Abstract resonator model}

The cavity segment $0<z<L$ consists of three parallel waveguides with a possibly wider, multimode central core. Restricting to the propagation of confined waves, the guided modes of the isolated port waveguides (one field per core for fixed light polarization) and of the isolated cavity core (possibly several modes) establish an approximate basis for the further modeling. Bidirectional copies of these profiles have to be taken into account; the total optical field inside the cavity segment becomes a superposition of the basis fields with separate amplitudes of forward and backward traveling modes, combined into amplitude vectors $\boldsymbol{F}$ and $\boldsymbol{B}$.

"Standard" coupled mode theory [29, 13], applied first to the forward wave propagation along the cavity segment, leads to a linear relation between the mode amplitudes $\boldsymbol{F}(L)$ at the end $z=L$ of the cavity and the amplitudes $\boldsymbol{F}(0)$ at the beginning $z=0$, with 
a propagation matrix $T$ that is given by the solutions of the coupled mode equations. For the present linear, nonmagnetic materials, the same matrix connects the amplitudes of the backward propagating waves:

$$
\boldsymbol{F}(L)=\mathrm{T} \boldsymbol{F}(0), \quad \boldsymbol{B}(0)=\mathrm{T} \boldsymbol{B}(L) .
$$

According to the above assumptions, the cavity facets have negligible influence on the basis fields in the port waveguides. Upon incidence onto the facets, parts of the optical power that is contained in the cavity modes is transmitted or reflected into nonconfined fields, i.e. lost for the field built up in the resonator. The remainder is reflected into a superposition of conversely propagating guided cavity modes, prospectively with altered relative amplitudes. Only this latter part is relevant for the resonator model. Hence, to describe the effect of the cavity facets, the amplitude vectors are split into a first part related to the port waveguides, and a second part that covers the amplitudes of the cavity modes. The cavity propagation matrix is divided into corresponding blocks as well,

$$
\boldsymbol{F}=\left(\begin{array}{c}
\boldsymbol{F}_{\mathrm{p}} \\
\boldsymbol{F}_{\mathrm{c}}
\end{array}\right), \quad \boldsymbol{B}=\left(\begin{array}{c}
\boldsymbol{B}_{\mathrm{p}} \\
\boldsymbol{B}_{\mathrm{c}}
\end{array}\right), \quad \mathrm{T}=\left(\begin{array}{cc}
\mathrm{T}_{\mathrm{pp}} & \mathrm{T}_{\mathrm{pc}} \\
\mathrm{T}_{\mathrm{cp}} & \mathrm{T}_{\mathrm{cc}}
\end{array}\right)
$$

where the indices $\mathrm{p}$ and $\mathrm{c}$ indicate port and cavity related entries. Now the result of the guided wave reflections at the (identical) facets can be stated as

$$
\boldsymbol{B}_{\mathrm{c}}(L)=\mathrm{R} \boldsymbol{F}_{\mathrm{c}}(L), \quad \boldsymbol{F}_{\mathrm{c}}(0)=\mathrm{R} \boldsymbol{B}_{\mathrm{c}}(0),
$$

with a symmetric reflectivity matrix $R$, that relates the incident mode amplitudes and the guided reflected waves.

For the present linear device with twofold symmetry, it is sufficient to consider optical input in only one of the external ports. Assigning indices 1 and 2 to the lower and upper waveguides, the external input amplitudes $\boldsymbol{F}_{\mathrm{p}}(0)=(1,0)^{\mathrm{T}}, \boldsymbol{B}_{\mathrm{p}}(L)=(0,0)^{\mathrm{T}}$ specify a unit excitation in the lower left port A. Then Eqs. (36), (37), and (38) predict the external output amplitudes

$$
\boldsymbol{F}_{\mathrm{p}}(L)=\left(\mathrm{T}_{\mathrm{pp}}+\mathrm{T}_{\mathrm{pc}} \mathrm{R} \Omega^{-1} \mathrm{~T}_{\mathrm{cc}} \mathrm{R} \mathrm{T}_{\mathrm{cp}}\right) \boldsymbol{F}_{\mathrm{p}}(0), \quad \boldsymbol{B}_{\mathrm{p}}(0)=\mathrm{T}_{\mathrm{pc}} \mathrm{R} \Omega^{-1} \mathrm{~T}_{\mathrm{cp}} \boldsymbol{F}_{\mathrm{p}}(0)
$$

and a field with amplitudes

$$
\boldsymbol{F}_{\mathrm{c}}(L)=\Omega^{-1} \mathrm{~T}_{\mathrm{cp}} \boldsymbol{F}_{\mathrm{p}}(0), \quad \boldsymbol{B}_{\mathrm{c}}(0)=\Omega^{-1} \mathrm{~T}_{\mathrm{cc}} \mathrm{R} \mathrm{T}_{\mathrm{cp}} \boldsymbol{F}_{\mathrm{p}}(0) .
$$

inside the cavity, with a resonance denominator $\Omega=1-\mathrm{T}_{\mathrm{cc}} \mathrm{RT} \mathrm{T}_{\mathrm{cc}} \mathrm{R}$ in matrix form. With the above numbering of the port waveguides, one obtains $P_{\mathrm{A}}=\left|B_{\mathrm{p}, 1}(0)\right|^{2}, P_{\mathrm{B}}=$ $\left|F_{\mathrm{p}, 1}(L)\right|^{2}, P_{\mathrm{C}}=\left|F_{\mathrm{p}, 2}(L)\right|^{2}, P_{\mathrm{D}}=\left|B_{\mathrm{p}, 2}(0)\right|^{2}$ for the relative fractions of guided power that are reflected into port $\mathrm{A}$, transmitted to $\mathrm{B}$, dropped forward to $\mathrm{C}$ or dropped backward into port D. For a detailed account of the present model we refer to Ref. [13].

Figure 11 shows an example for a spectral response that has been computed using these equations, evaluated for a dense series of wavelengths. Apart from the approximation of the cavity segment by coupled mode theory, the simulations are based on a rigorous facet model (bidirectional eigenmode propagation, BEP). The results as shown agree reasonably with rigorous BEP simulations of the entire resonator device [13]. 

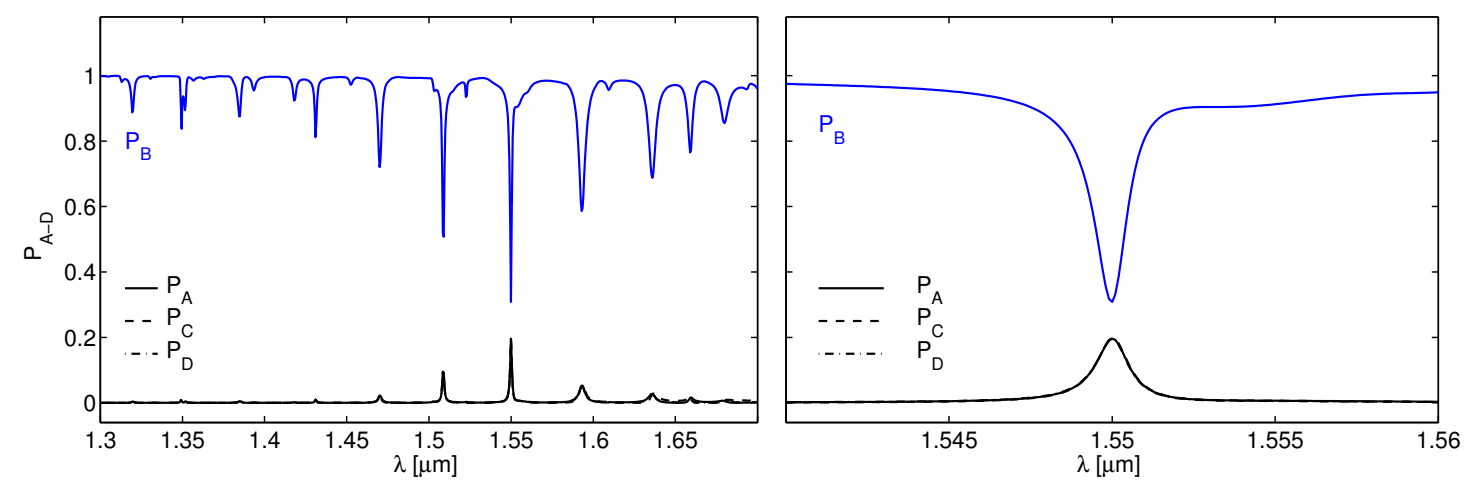

FIGURE 11. Spectral response of a resonator device according to Figure 10 with dimensions $L=$ $5.738 \mu \mathrm{m}, W=2.524 \mu \mathrm{m}, g=0.450 \mu \mathrm{m}, w=0.112 \mu \mathrm{m}$ for refractive indices $n_{\mathrm{b}}=1.45$ and $n_{\mathrm{g}}=3.40 ; 2 \mathrm{D}$ simulations for TE polarized fields. $P_{\mathrm{A}}$ to $P_{\mathrm{D}}$ are the relative power fractions that are reflected respectively transmitted into ports A to D.

The spectrum is rather irregular, with a pronounced resonance that is magnified in the right inset. For most wavelengths (off resonance), the input power is directly transferred, $P_{\mathrm{B}}$ is close to the unit level. Resonances appear as a drop in $P_{\mathrm{B}}$ and a simultaneous increase in $P_{\mathrm{A}}, P_{\mathrm{C}}, P_{\mathrm{D}}$, where the latter curves are partially superimposed in Figure 11 . In contrast to the ring resonators, the rectangular cavity effects an equal power drop in the forward and backward directions; in an extremal case a quarter of the input power would be scattered into all four output ports.

Figure 12 illustrates the field profile at resonance. One observes partly standing/traveling waves in port A, given by the superposition of the unit input and the less than quarter reflection, and outgoing traveling guided waves in ports B, C, and D. A purely standing wave is visible inside the cavity, with a characteristic pattern of nodal lines. In Section 3.2 we will try to explain that pattern.

So far the power transmission scheme is not directly useful for an application as an add-drop-filter, where one would prefer to have the scattered power concentrated into a single output port at resonance. This is indeed possible to realize by cascading of two cavities, such that the individual fields interact through the port waveguides and by direct evanescent coupling. With a suitable choice of the cavity distance, at resonance a standing wave pattern with alternating maxima in the two cavities establishes; apart from radiation losses the power is exclusively dropped in the forward drop channel (port D in Figure 10). See Refs. [30, 9, 10] for the abstract concept, for specific examples, and for detailed numerical experiments on these filters.

\subsection{Rectangular resonances}

A characterization of resonant configurations is possible in a way quite analogous to Section 2.1. Resonances manifest as singularities in the denominator $\Omega$ in Eqs. (39), (40). At resonance a field establishes inside the cavity with a mode amplitude vector that resembles an eigenvector corresponding to a large eigenvalue of $\Omega^{-1}$, to a zero eigenvalue of $\Omega$, or to a unit eigenvalue of $T_{c c} R T_{c c} R$, where the latter chain of operators 


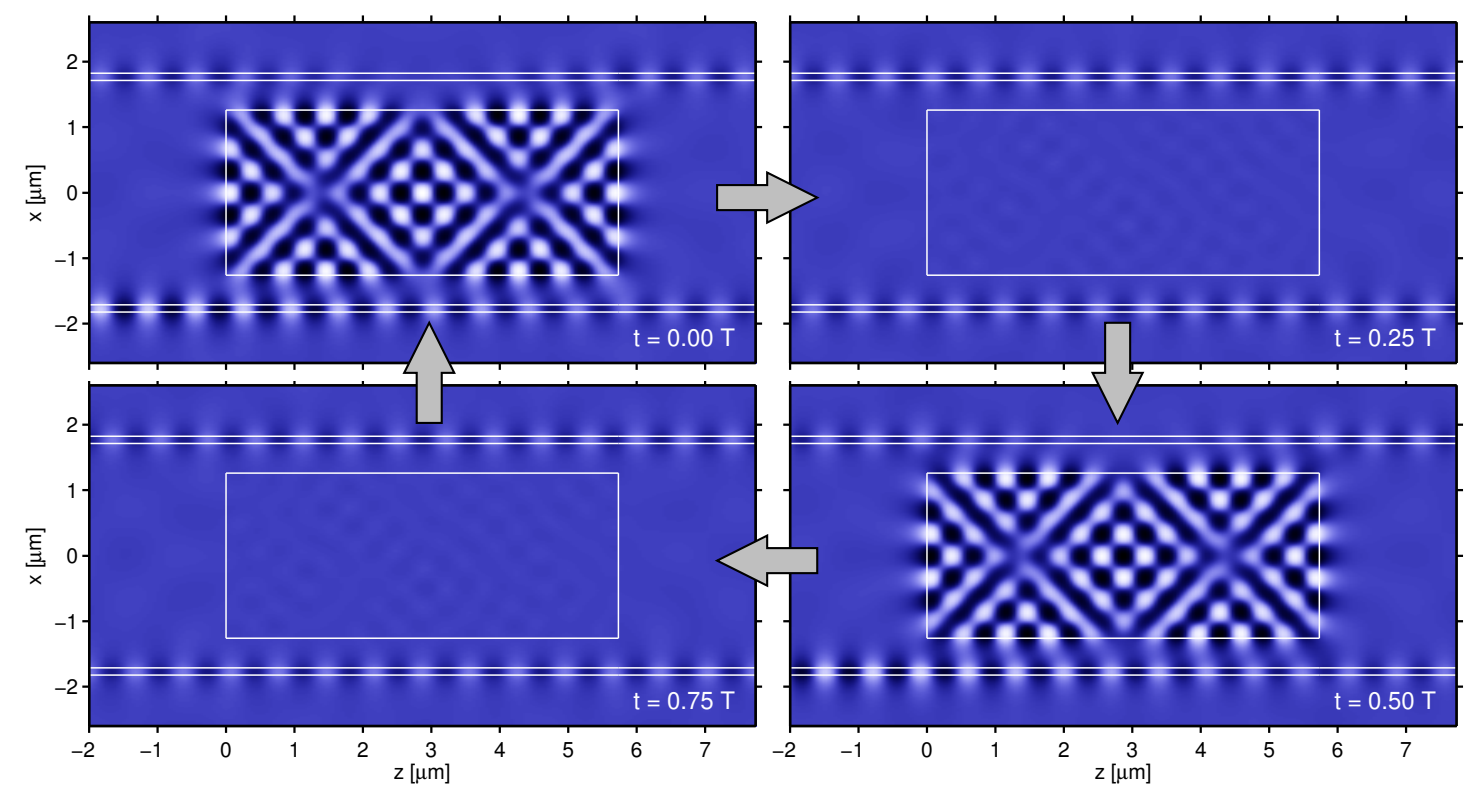

FIGURE 12. Field pattern for the pronounced resonance in Figure 11 at $\lambda=1.55 \mu \mathrm{m}$, extremal snapshots of the single electric $y$-component of the TE fields, at times $t$ equally distributed over one period $T$. The rigorous simulation from [13] predicts relative power transmissions of $P_{\mathrm{A}}=24.4 \%, P_{\mathrm{B}}=25.5 \%$, $P_{\mathrm{C}}=24.5 \%$, and $P_{\mathrm{D}}=24.2 \%$.

allows a quite descriptive interpretation: A field is excited at one end of the cavity that, after being reflected once, transferred through the cavity, reflected a second time, and being transferred back to its original position, resembles the initial field.

In the limit of a large gap width $g$, the cavity related block $T_{c c}$ of $T$ becomes a diagonal matrix with entries given by the phase evolutions of the individual cavity modes, and $\Omega$ becomes a property of the isolated cavity. A formal, quantitative evaluation of these notions in Ref. [13] shows a very good agreement with rigorous simulations. This leads to a classification of the various resonant peaks in plots like Figure 11 as singleor bimodal resonances, where one or two of the cavity modes with different orders contribute. The conclusion that a representation of resonant fields in the dielectric rectangles in terms of (few) slab waveguide modes is adequate motivates the following reasoning.

The isolated cavity as sketched in Figure 13 can be considered as a segment of a slab waveguide of thickness $W$. At resonance a guided mode with a specific propagation constant $\beta_{z}=k n_{\mathrm{g}} \cos \theta_{z}$ and an associated mode angle $\theta$ should fit into that slab, where the fields propagate in the positive and negative $z$-direction. Alternatively, the cavity can be regarded as a segment of a slab of thickness $L$, with modes propagating along the vertical $x$-axis. Also in this view the resonant field is constituted by a slab mode, now supported by the core of thickness $L$ with a propagation constant $\beta_{x}=k n_{\mathrm{g}} \cos \theta_{x}$. Certainly both descriptions must result in identical expressions for the field inside the cavity, i.e. the mode angles with respect to the respective propagation axes are related by $\theta_{x}=\pi / 2-\theta_{z}$.

Hence one can state the following characterization: A rectangular cavity of width $W$ 


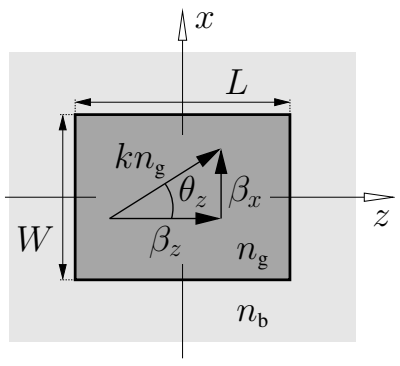

FIGURE 13. The isolated cavity of the resonator in Figure 10, to be viewed as a horizontal slab segment of thickness $W$ and length $L$, or alternatively as a vertical segment of thickness $L$ and length $W$.

and length $L$ shows a resonance at vacuum wavelength $\lambda$, if simultaneously the slab of thickness $W$ supports a mode with angle $\theta$ and the slab of thickness $L$ supports a mode with angle $\pi / 2-\theta$, both at wavelength $\lambda$ and for the symmetric refractive index profiles of the cavity. While this refers to a single mode resonance with a field based upon only one (forward and backward propagating) mode per axis, a bimodal resonance is characterized by the existence of a pair of modes per axis orientation that satisfy the phase relations simultaneously.

Resonant configurations, e.g. widths $W$ and lengths $L$ of dielectric rectangles that show a resonance with specific horizontal and vertical modal order at a given vacuum wavelength, can thus be found by looking at curve intersections in a plot of mode angles $\theta$ and $\pi / 2-\theta$ versus the thickness of a corresponding slab waveguide. A more formal treatment of this argument and further examples can be found in Ref. [13].

\subsection{Multimode reflection at a slab waveguide facet}

According to the former quantitative characterization in terms of amplification factors based on eigenvalues of $\Omega$, high quality resonances in a dielectric rectangle require a facet reflectivity close to unity for the contributing cavity modes. Hence we close this paper with a look to what happens around the facet of the cavity core. Figure 14 introduces the facet geometry.

We refer to the common ray model for confined wave propagation in 2D slab waveguides. Via the relation $\beta=k n_{\mathrm{g}} \cos \theta$ each mode has a mode angle $\theta$ associated with its propagation constant $\beta$. This angle may alternatively be interpreted as an incidence angle of the waves on the facet plane. A high reflectivity can be expected if that incidence angle is larger than the angle $\theta_{\text {crit }}$ for total plane wave reflection at the facet interface, with $\sin \theta_{\text {crit }}=n_{\mathrm{b}} / n_{\mathrm{g}}$. The maximum angle $\theta_{\max }$ for guided wave propagation in a symmetric slab with refractive indices $n_{\mathrm{b}}, n_{\mathrm{g}}$ is given by $\sin ^{2} \theta_{\max }=1-n_{\mathrm{b}}^{2} / n_{\mathrm{g}}^{2}$. Guided waves with angles in the interval $\theta_{\text {crit }}<\theta_{\max }$ should exist. Consequently one obtains a lower limit $n_{\mathrm{g}}>\sqrt{2} n_{\mathrm{b}}$ for the refractive index contrast that is necessary to realize a high reflectivity facet.

As as example, Figure 15 shows the reflectivity at the facet of a thick multimode waveguide with large refractive index contrast. Several higher order modes are supported with angles between $\theta_{\text {crit }}$ and $\theta_{\text {max }}$; indeed a large single mode reflectivity can be observed for these fields. Nevertheless, the maximum levels of about $80 \%$ are by far not sufficient to explain the high quality resonances in Figure 11. 


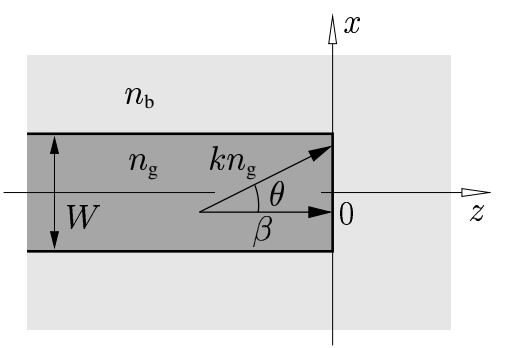

FIGURE 14. A facet of the (multimode) waveguide that forms the resonator cavity in Figure 10. Each mode has assigned a propagation constant $\beta$ and propagation angle $\theta$.

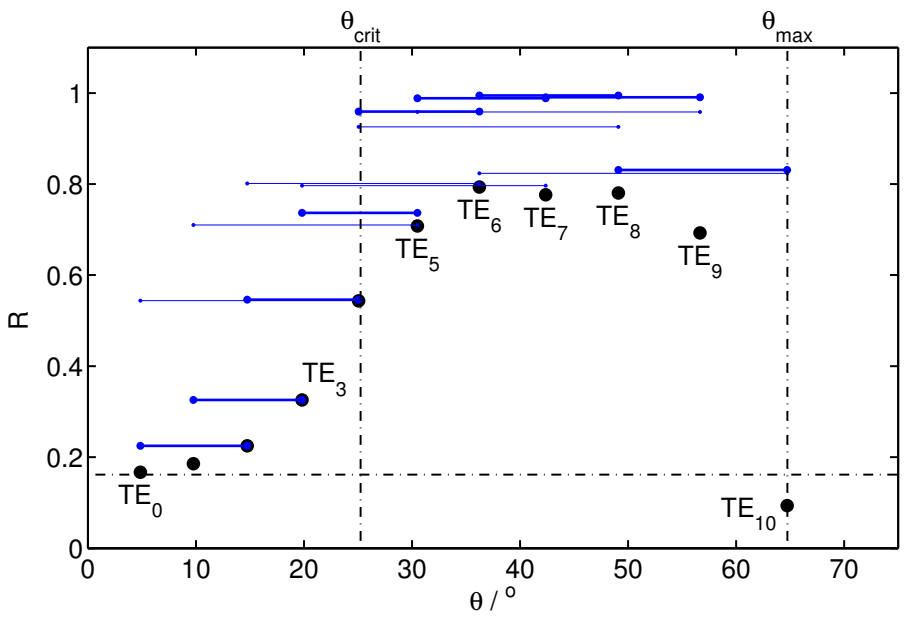

FIGURE 15. Relative reflected guided powers $R$ of single modes (filled circles) and of two mode superpositions (bars) versus the discrete mode angle, for a waveguide facet with the parameters of the cavity of Figure 12; BEP simulations from Ref. [13]. The relative amplitudes for the superpositions are adjusted such that the amount of power that is reflected into the incoming modes is maximized in each case.

The picture changes dramatically, if more than two modes with equal symmetry are incident on the facet plane simultaneously. The bars in Figure 15 indicate the maximum amount of power that is reflected back into a superposition of the two modes indicated by the bar end points, with suitable relative input amplitudes. The simulation predicts a highest value of $99.4 \%$ reflected power for the modes $\mathrm{TE}_{6}$ and $\mathrm{TE}_{8}$, with the difference to full reflection being beyond the accuracy that can be expected from the numerical computations. The origin of this raise towards almost total reflection is an interference effect, as revealed by inspecting the corresponding field patterns in Figure 16.

One observes mainly standing waves with a checkered extremal pattern inside the core for incidence of the individual modes $\mathrm{TE}_{6}$ and $\mathrm{TE}_{8}$, with exponential mode tails at the upper and lower waveguide interface, and a similar field decay across the facet. The radiation outside the core appears as two cylindrical waves, originating from the edges in the facet plane. If the relative amplitude and phase of the two modes are adjusted such that their superposition vanishes in these points, the cylindrical waves found for the single mode incidences interfere destructively; almost the entire incident power is then reflected. The very high level of more than $99 \%$ reflection and the agreement of the resulting field profile with the wave pattern inside the resonator cavity in Figure 12 indicates that this is indeed the mechanism that enables the bimodal resonances. For a more detailed investigation of this total reflection phenomenon we refer to Ref. [31]. 

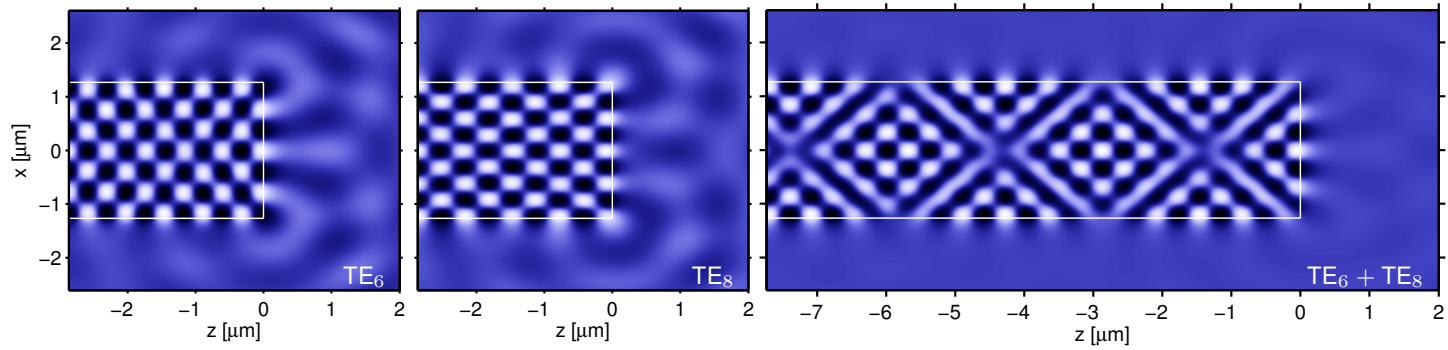

FIGURE 16. Extremal field profiles around the facet of a multimode waveguide with the parameters of the cavity of Figure 12, for an excitation by individual modes of order 6 (left) and 8 (center), and for a specific superposition of these two incoming fields (right). Rigorous BEP simulations [13] predict relative power fractions of $79 \%\left(\mathrm{TE}_{6}\right), 78 \%\left(\mathrm{TE}_{6}\right)$, and $99.3 \%\left(\mathrm{TE}_{6}+\mathrm{TE}_{8}\right)$ that are reflected into the incoming fields.

\section{CONCLUDING REMARKS}

Sections $2.1-2.3$ establish a complete quasi-analytical ab-initio model for 2D optical ring resonators. Together with the dependence of the cavity attenuation on the ring radius (e.g. Figure 6) and the performance diagrams in Figure 3, plots like those shown in Figure 9 allow to select all geometrical dimensions that enter the present ring resonator design. If suitable basis fields would be available - mode profiles for 3D straight and bent dielectric channel waveguides with $2 \mathrm{D}$ cross section - extension of the present formalism to realistic 3D simulations should be straightforward. Also the extension to more general cases, e.g. resonators with nonidentical couplers, or cavities where more than one mode plays a role (polarization issues, higher order gallery modes) should pose no principal problems, though the equations of Section 2.1 would have to be revised.

The concepts concerning rectangular optical microresonators are certainly speculative; currently only 2D models exist. A variant as proposed in Ref. [32] could be somewhat more realistic, where waveguide Bragg gratings constitute the cavity reflectors. Entirely different regimes of parameters apply, with significantly lower refractive index contrast at the cost of an increased device length. The device is an example where a description in terms of coupled mode theory for the coupling segment and the Bragg gratings, embedded in the framework of Section 3.1, works surprisingly well. Only single mode resonances are relevant, the filter concept can be applied. Problems for a real device could arise from out-of-plane losses, which are not covered in the present models.

Perhaps the standing wave phenomena as shown can be viewed as a limit of what could also be expected from a circular, ring- or disc-shaped cavity in case of small cavity diameters, where the refractive index variations along the light paths can no longer be considered as adiabatic, such that reflections will become relevant.

\section{ACKNOWLEDGMENTS}

Financial support by the European Commission (project IST-2000-28018, 'NAIS') is gratefully acknowledged. The authors would like to thank E. van Groesen, H. J. W. M. Hoekstra and in particular the colleagues within the NAIS project for many fruitful discussions on the subject. 


\section{REFERENCES}

1. Driessen, A., Geuzebroek, D., Hoekstra, H. J. W. M., Kelderman, H., Klein, E. J., Klunder, D. J. W., Roeloffzen, C. G. H., Tan, F. S., Krioukov, E., Otto, C., Gersen, H., van Hulst, N. F., and Kuipers, L., Microresonators as building blocks for VLSI photonics (2004), this volume.

2. NAIS, Next-generation active integrated optic subsystems (project start: 2001), Information society technologies programme of the European Commission, project IST-2000-28018, http://www.mesaplus.utwente.nl/nais/.

3. Yariv, A., Electronic Letters, 36, 321-322 (2000).

4. Okamoto, K., Fundamentals of Optical Waveguides, Academic Press, SanDiego, 2000.

5. Čtyroký, J., Prkna, L., and Hubálek, M., Guided-wave optical microresonators: Calculation of eigenmodes (2004), this volume.

6. Marcatili, E. A. J., The Bell System Technical Journal, September, 2103-2132 (1969).

7. Nöckel, J. U., and Stone, A. D., Nature, 385, 45-47 (1997).

8. Gornik, E., Science, 280, 1544-1545 (1998).

9. Manolatou, C., Khan, M. J., Fan, S., Villeneuve, P. R., Haus, H. A., and Joannopoulos, J. D., IEEE Journal of Quantum Electronics, 35, 1322-1331 (1999).

10. Lohmeyer, M., Optical and Quantum Electronics, 34, 541-557 (2002).

11. Nosich, A. I., and Boriskina, S. V., Proceedings of the International Conference on Transparent Optical Networks (ICTON 2000), Gdansk, Poland, pp. 27-30 (2000).

12. Boriskina, S. V., Benson, T. M., Sewell, P., and Nosich, A. I., Optical and Quantum Electronics, 35, 545-559 (2003).

13. Hammer, M., Optics Communications, 214, 155-170 (2002).

14. Stoffer, R., Hiremath, K. R., and Hammer, M., Comparison of coupled mode theory and FDTD simulations of coupling between bent and straight optical waveguides (2004), this volume.

15. Vassallo, C., Optical Waveguide Concepts, Elsevier, Amsterdam, 1991.

16. Lohmeyer, M., Bahlmann, N., and Hertel, P., Optics Communications, 163, 86-94 (1999).

17. Pennings, E. C. M., Bends in optical ridge waveguides, Delft University of Technology, Delft, The Netherlands, 1990, Ph.D. Thesis.

18. Hiremath, K. R., Modeling of 2D cylindrical optical microresonators (2003), MSc Thesis.

19. Hall, D. G., and Thompson, B. J., editors, Selected Papers on Coupled-Mode Theory in GuidedWave Optics, vol. MS 84 of SPIE Milestone Series, SPIE Optical Engineering Press, Bellingham, Washington USA, 1993.

20. Marcuse, D., Theory of Dielectrical Optical Waveguides, 2nd edition, Academic Press, London, 1991.

21. Rowland, D. R., and Love, J. D., IEE Proceedings, Pt. J, 140, 177-188 (1993).

22. Little, B. E., Chu, S. T., Haus, H. A., Foresi, J., and Laine, J.-P., Journal of Lightwave Technology, 15, 998-1005 (1997).

23. Chin, M. K., and Ho, S. T., Journal of Lightwave Technology, 16, 1433-1446 (1997).

24. Boriskina, S. V., and Nosich, A. I., IEEE Transactions on Microwave Theory and Techniques, 47, 224-231 (1999).

25. Little, B. E., Laine, J.-P., and Haus, H. A., Journal of Lightwave Technology, 17, 704-715 (1999).

26. Klunder, D. J. W., Krioukov, E., Tan, F. S., van der Veen, T., Bulthuis, H. F., Sengo, G., Otto, C., Hoekstra, H. J. W. M., and Driessen, A., Applied Physics B, 73, 603-608 (2001).

27. Klunder, D. J. W., Tan, F. S., van der Veen, T., Bulthuis, H. F., Sengo, G., Docter, B., Hoekstra, H. J. W. M., and Driessen, A., Journal of Lightwave Technology, 21, 1099-1110 (2003).

28. Chuang, S. L., Journal of Lightwave Technology, 5, 5-15 (1987).

29. Lohmeyer, M., Bahlmann, N., Zhuromskyy, O., and Hertel, P., Optical and Quantum Electronics, 31, 877-891 (1999).

30. Fan, S., Villeneuve, P. R., Joannopoulos, J. D., and Haus, H. A., Physical Review Letters, 80, 960-963 (1998).

31. Hammer, M., and van Groesen, E., Journal of Lightwave Technology, 20, 1549-1555 (2002).

32. Hammer, M., Yudistira, D., and Stoffer, R., "Modeling of grating assisted standing wave microresonators for filter applications in integrated optics", Optical and Quantum Electronics (2004), accepted for publication. 